International Journal of Integrative Pediatrics and Environmental Medicine, V5, 2019, 16-19

\title{
A NEWBORN INFANT WITH TACHYPNEA
}

\author{
Linh Ha MD ${ }^{1}$, Tetyana L. Vasylyeva MD PhD ${ }^{1^{*}}$
}

Author information: ${ }^{1}$ Department of Pediatrics, Texas Tech University Health Sciences Center; 1400 South Coulter Street, Amarillo, TX 79106; email: Tetyana.vasylyeva@ttuhsc.edu

\begin{abstract}
This article is a brief review of a newborn infant with Joubert Syndrome (JS). The patient's presentation was characterized by persistent episodic tachypnea in the newborn period, which eventually lead to the diagnosis. The JS has multiorgan involvement with major impacts on the brain and the kidney function. The early recognition of this rare disorder has the potential to affect treatment and outcome. Although rare, recognition of genetic disorders in babies at birth is important to direct initial and continued pediatric care. The management of JS requires a multidisciplinary team, including primary care and multiple subspecialists (in this case- nephrology and neurology).
\end{abstract}

Key words: Jourbert syndrome (JS), newborn, tachypnea.

\section{CASE}

A $4170 \mathrm{~g}$ male infant was delivered by elective caesarean section at $403 / 7$ weeks of gestation to a healthy gravida 1 mother from a small Guatemalan village. APGAR scores of 7 and 8 , at 1 and 5 minutes, respectively, were observed. Twelve hours after birth the infant developed an irregular breathing pattern with episodic tachypnea and a respiratory rate up to 100 breaths per minute. On physical exam, the patient displayed general hypotonia, horizontal nystagmus, ptosis of the right eye, a trapezoidshaped mouth, tongue protrusion, posteriorly rotated large ears, and bilateral single palmar creases (Figure 1-4). Abdominal exam revealed bilateral palpable enlarged kidneys.

Chest x-ray and serum chemistries were normal, except for a mildly depressed $\mathrm{CO}_{2}$ level of 19 $\mathrm{mmol} / \mathrm{l}$, an elevated creatinine of $1.43 \mathrm{mg} / \mathrm{dl}$.

*Corresponding author: Tetyana L. Vasylyeva, Department of Pediatrics, Texas Tech University Health Sciences Center; 1400 South Coulter Street, Amarillo, TX 79106; email: Tetyana.vasylyeva@ttuhsc.edu

L. Ha, TL. Vasylyeva (
Abdominal ultrasound revealed large kidneys without cortico-medullar differentiation containing cysts of varying sizes. An MRI of the brain, performed at 25 days of age, revealed dilatation of the fourth ventricle, a small cerebellar vermis, an enlarged posterior fossa, and a molar tooth sign (deep interpeduncular fossa, narrow isthmic region, thickened superior cerebellar peduncles, hypoplastic cerebellar vermis) (Figure 5,6). Parents denied consanguinity and any family history of genetic abnormalities. The baby's karyotype was $46 \mathrm{XY}$ and constellation of symptoms led to a diagnose of JS.

\section{DISCUSSION}

Jourbert Syndrome was first described in 1969 by Maria Joubert, a pediatric resident, as a familial syndrome in 4 Canadian siblings [1]. She noted episodic hyperpnea, abnormal eye movement, ataxia, and retardation, with hypoplasia of the cerebellar vermis. JS has been diagnosed in approximately 350 cases worldwide. It is an autosomal recessive disorder characterized by the complete or partial agenesis of the cerebellar vermis $[1,2]$. Its prevalence in the U.S. has been estimated at 1:1,000,000 [3]. JS 
belongs to a group of cerebello-oculo-renal syndromes and can be a diagnostic challenge to neonatologists and general pediatricians because of its rarity. abnormalities (episodic tachypnea/hyperpnea), ocular and oculomotor findings (ptosis and ocular motor apraxia), renal anomaly (cystic kidney disease), and additional pathologies (congenital

Three-month-old child with Joubert Syndrome

Figure 1. Bilateral post-rotated large ear/

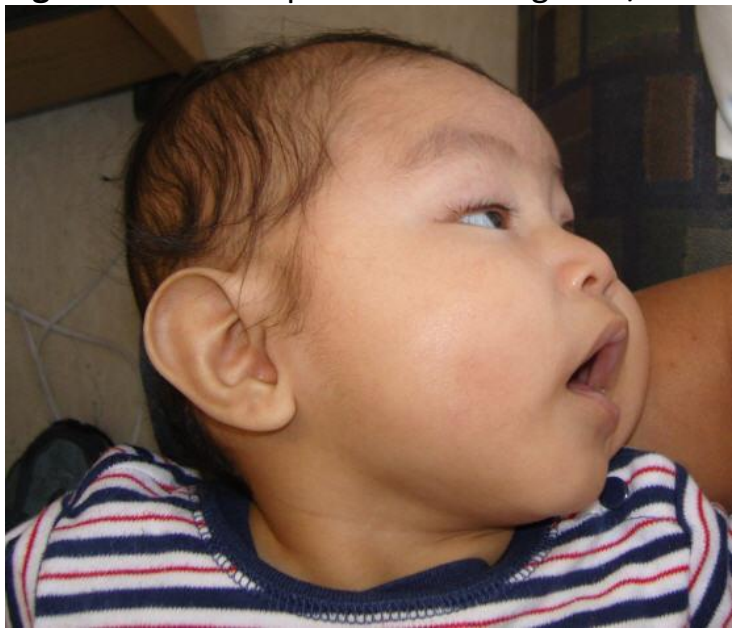

Figure 3. Trapezoid-shaped mouth and tongue protrusion

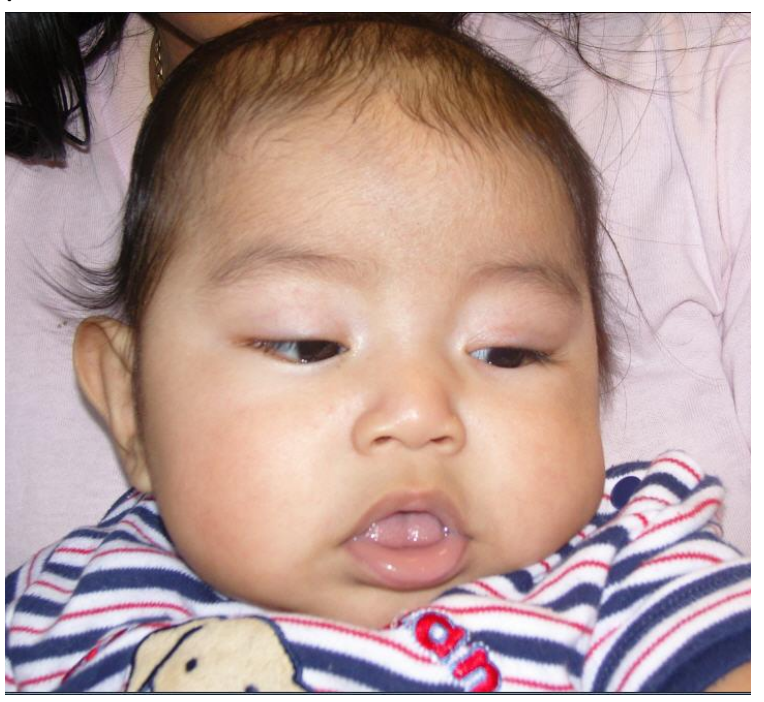

Figure 2. Ptosis of the right eye.

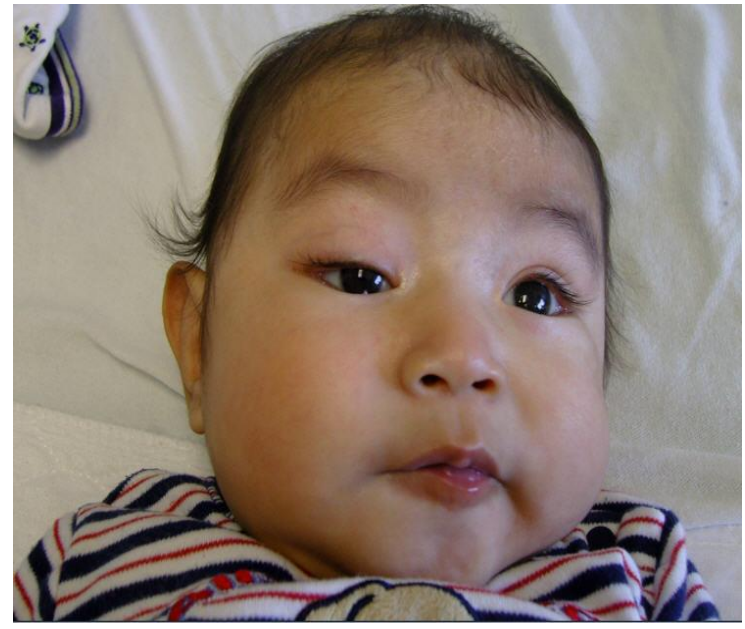

Figure 4. Bilateral, single transverse palmar crease

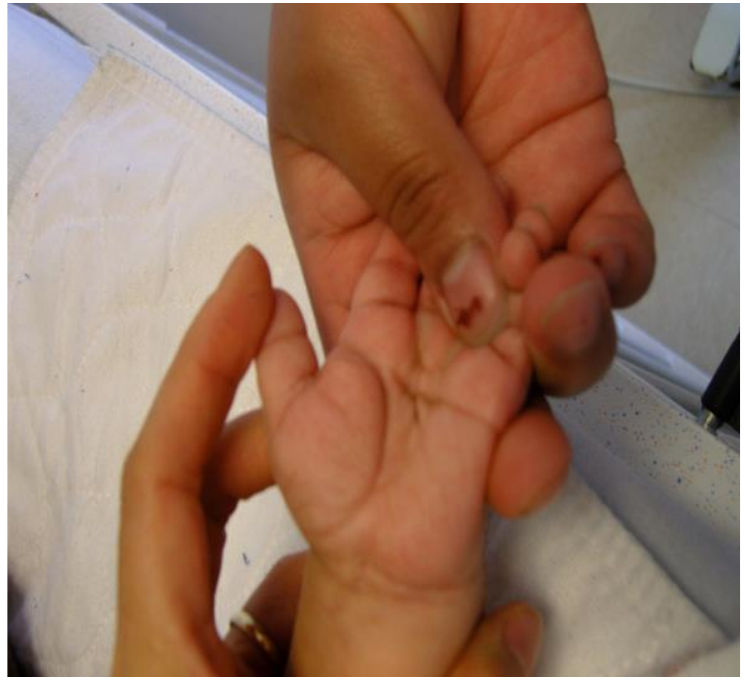

The core diagnostic criteria to diagnose Joubert's Syndrome includes CNS features (hypotonia, ataxia, and global developmental delays), neuroimaging (molar tooth sign), respiratory heart disease, ocular coloboma, progressive retinal dysplasia, hepatic fibrosis, hydrocephalus, occipital encephalocele, polydactyly, and tongue protrusion) [4]. 
Figure 5. Axial T1-weighted MRI at the level of the brainstem reveals molar tooth sign.

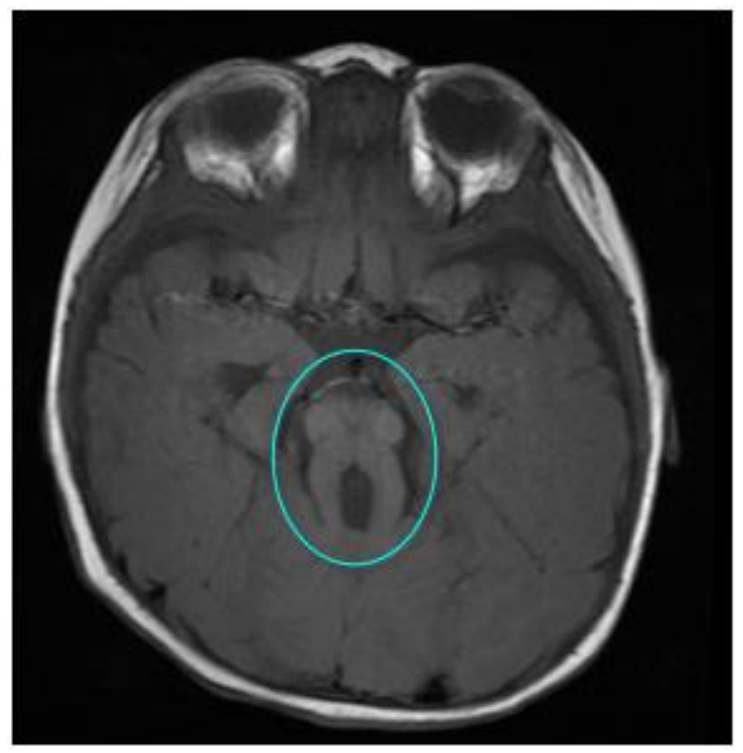

Figure 6. Sagital T1-Weighted MRI: Vermis (v) dysplasia, ventricular enlargement of fourth ventricle and elevated roof (arrow).

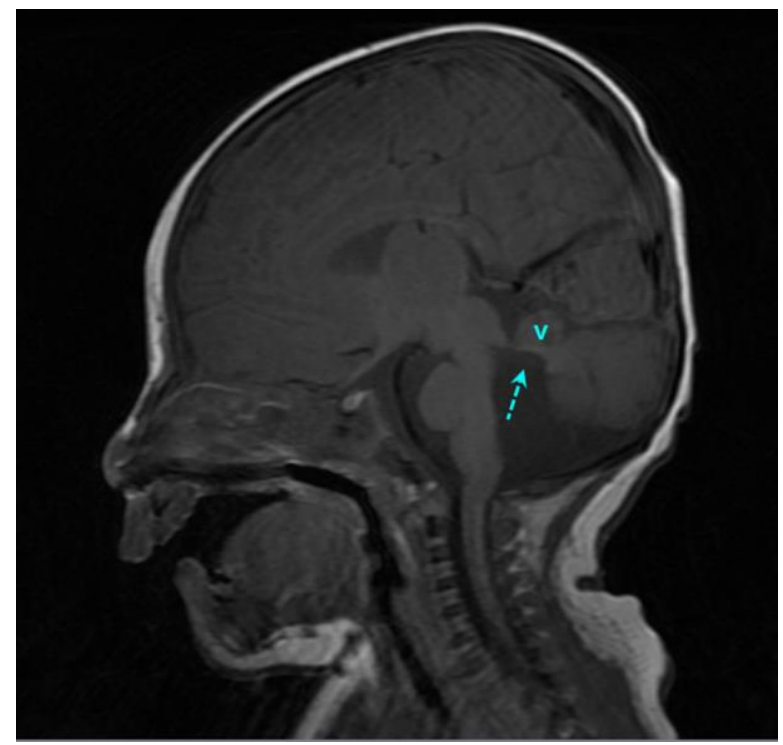

To date, five genes have been linked to JS: AHI1 (6q23.3), NPHP1 (2q13), CEP290/NPHP6 (12q21.3), MKS3 (8q22.1), and RPGRIP1L (16q12.2). The involvement of numerous chromosomes and a large variety of associated abnormalities suggest significant genetic heterogeneity in this syndrome $[4,5]$

The radiologic hallmark of JS is malformation of the cerebellum (aplasia of the vermis) and the brainstem (molar tooth sign). The breathing pattern abnormalities (present in over $40 \%$ of patients) may be explained by the dysplastic caudal medulla. Oculomotor disturbances are found in $42-77 \%$ of children with JS. Renal involvement has been described in 30\% of patients and may take the form of cystic dysplasia (as seen in our patient) or juvenile nephronopthisis [6]. Studies have shown strong associations between retinal and renal manifestations [3].

It is difficult to distinguish JS from other related syndromes such as: Dandy-Walker syndrome, congenital disorders of glycolysation; and cerebellar vermis hypoplasia, oligophrenia, ataxia, coloboma, hepatic fibrosis $(\mathrm{COACH})$ syndrome. These disorders have vermis hypoplasia, olivopontocerebellar atrophy, eye movement abnormalities and ataxia, but none of these disorders has abnormal breathing patterns. Children with JS display a wide range of disabilities. Infant apnea, visual and renal insufficiency, motor dysfunction, speech difficulties, cognitive deficits, and behavioral problems are prominent with approximately $30 \%$ of patients dying in infancy secondary to respiratory complications [8]. Patients with JS are extremely sensitive to the respiratory depressant effects of anesthetic agents. Opioids and nitrous oxide should be avoided, and respiratory efforts should be closely monitored during any clinical procedures [9].

Once a diagnosis of JS is made, genetic counseling 
is important to ensure future siblings can be diagnosed antenatally via ultrasonagraphy, by searching for abnormal findings in the fetal posterior fossa and kidneys during subsequent pregnancies [7]. This infant presented with typical characteristics of JS with cerebellar, ocular, respiratory, and renal findings. The baby was monitored for hyperpnea, metabolic acidosis, azotemia, and hypertension. He was discharged to home from the hospital at 1month-old, on captopril and sodium citrate (Bicitra), with close follow-up scheduled with his pediatrician and sub-specialists (neurology, ophthalmology, nephrology and others). He presented at follow up in clinic with hypotonia and severe global developmental delay. At 9months of age, he demonstrated a social smile, continued head lag, and retained an inability to roll over or babble. The management of JS is multidisciplinary and supportive. It requires a general pediatrician, ophthalmologist, geneticist, nephrologist, neurologist, behaviordevelopmental specialist, and physical/occupational/speech therapists to ensure that JS children have adequate support and treatment from multidisciplinary team to address global development and symptomatic treatment

\section{REFERENCES}

1. Jourbert M, Eisenring JJ, Robb JP, Andermann F. Familial agenesis of the cerebellar vermis: A syndrome of episodic hyperpnea, abnormal eye movements, ataxia, and retardation. J Child Neurol. 1969; 19:813-825. DOI:10.1177/088307389901400902
2. Salman MS, Maria BL. In: Maria BL. Current Management in Child Neurology. Shelton, CT: People's Medical Publishing House; 2009:576-577.

3. Parisi MA, Doherty D, Chance PF, Glass TA. Joubert syndrome and related disorders. Eur J Hum Genet. 2007;15:511-521. DOI:10.1038/sj.ejhg. 5201648

4. Maria BL, Boltshauser E, Palmer SC, Tran TX. Clinical features and revised diagnostic criteria in Joubert syndrome. J Child Neurol. 1999;14:583. DOI: $10.1177 / 088307389901400906$

5. Harris PC. Genetic complexity in Joubert syndrome and related disorders. Kidney Int. 2007;72:1421-1423. DOI: 10.1038/sj.ki.5002577

6. Sampathkumar K, Sooraj YS, Karunakaran N, Ganesh R, Mahaldar AR. Joubert syndrome. Kidney Int. 2008;74:1222. DOI:10.1038/ki.2008.339

7. Ní Scanaill S, Crowley P, Hogan M, Stuart B. Abnormal prenatal sonographic findings in the posterior cranial fossa: a case of Joubert's syndrome. Ultrasound Obstet Gynecol. 1999;13(1):71-4. DOI:10.1046/j.14690705.1999.13010071.x

8. Hodgkins PR, Harris CM, Shawkat FS, Thompson DA, Chong K, Timms C, RussellEggitt I, Taylor DS, Kriss A. Joubert syndrome: long term follow up. Dev Med Child Neurol. 2004;46(10):694-699.

9. Van Beek EJ, Majoie CB. Case 25: Joubert syndrome. Radiology. 2000;216(2):379-382. DOI: 10.1148/radiology.215.1.r00ap23235 